\title{
A sideways glance: Lamarck strikes back? Fathers pass on to progeny characteristics they develop during their lives
}

\author{
Sancia Gaetani
}

Received: 21 March 2011/Accepted: 19 June 2012/Published online: 5 July 2012

(C) Springer-Verlag 2012

The global prevalence of obesity is increasing across all age groups and in both sexes, contributing to the early emergence of insulin resistance usually followed by the development of type 2 diabetes (Kahn and Flier 2000; Reaven 1995). The pathology is determined by resistance to insulin action in multiple tissues, accompanied by failure of pancreatic $\beta$-cells to compensate the lack of response through increased insulin synthesis and secretion, a condition that leads to $\beta$-cell damage (Graham and Kahn 2007). Obesity in humans appears to be mostly related to complex interactions between genetic and environmental factors. Although diet affects the function of several somatic cell types playing a key role also in diseases like obesity and diabetes, it is widely accepted that none of the somatic cell effects can be transmitted to the next generation. The majority of environmental factors does not alter the animal's DNA sequence, but rather promote alterations that can influence somatic cell metabolism, and therefore the health status of the individual exposed to them. In the current paradigm for disease etiology, either single-nucleotide polymorphisms (SNiPs) or chromosomal abnormalities can promote the onset of a disease; however, evidences are accumulating in the last years that also the environment is equally important in disease etiology.

Diet and food components are the prime environmental factors that affect the animal genome, transcriptome, proteome, and metabolome, and this life-long interaction largely defines the health or disease state of any individual. Most, if not all, nutrients have at least indirect effects on gene and protein expression and therefore on metabolism.

S. Gaetani $(\square)$

INRAN, Via Ardeatina 546, 00178 Rome, Italy

e-mail: sancia.gaetani@gmail.com
The genome is evolutionarily and chemically rather stable. The ability of environmental factors to influence or promote disease onset and progression does not generally involve induction of DNA mutations, but acts through the regulation of genome activity, influencing disease etiology at different stages of development. Such altered metabolic programs can in turn promote abnormal physiology and disease onset at later developmental stages through epigenetic mechanisms.

The past few years have witnessed an important expansion in the understanding of inheritance, in parallel with the increasing variety of epigenetically inherited traits that have been described. Mechanisms thus exist that could allow organisms to tell their progeny about prevailing environmental conditions like for example the repeated exposure to a moderately toxic environment. Whether or not organisms can inherit characters induced by ancestral environments has serious implications. This type of inheritance is called "Lamarckian" inheritance after JB Lamarck who believed in the inheritance of acquired traits.

The term "epigenetics" was coined by Conrad Waddington in the 1940s to integrate the concepts of embryological growth and differentiation (epigenesis) with genetics and has evolved since then with the increased understanding of genes and genomes. Epigenetics focuses on molecular elements that can influence chromatin structure, irrespective of the DNA sequence. At present, the best definitions of epigenetics seem to be: "molecular factors and processes around DNA that are mitotically stable and regulate genome activity independently from DNA sequence" or "the study of mitotically and/or meiotically heritable changes in gene expression or cellular phenotype, which occur without changes to the underlying DNA sequence." The first epigenetic molecular factor, identified in the 1970s, was DNA methylation. In the 
mid-1990s, DNA methylation was followed by histone modification, together with the appreciation of the role of chromatin structure in genome regulation. Then, around the year 2000, non-coding RNA was discovered, and finally in 2005 , the first whole epigenome was analyzed. New epigenetic processes have been described in the past few years, whose function is not yet completely understood.

An important implication of epigenetic inheritance systems is that they provide a potential mechanism by which parents could transfer information to their offspring about the environment they experienced. The ability of environmental factors to promote a phenotype or a disease state not only in the exposed individuals but also in subsequent progeny for successive generations is termed transgenerational inheritance. The majority of environmental factors such as nutrition does not promote genetic mutations or alterations in DNA sequence but have the capacity to alter the epigenome. Germline epimutations that become permanently programmed allow transmission of epigenetic transgenerational phenotypes and promote disease.

Environmental factors could cause epigenetic modifications of somatic cells in individuals exposed to them $\left(\mathrm{F}_{0}\right.$ generation). In pregnant females $\left(\mathrm{F}_{0}\right)$, environmental exposure resulting in epigenetic modifications can lead to the transmission of epigenetic changes to the following 2 generations (fetus somatic cells, $\mathrm{F}_{1}$, and germ line cells of the fetus, effect on $\mathrm{F}_{2}$ ). The effect of this multigenerational exposure in subsequent generations ( $\mathrm{F}_{3}$ and beyond) is considered a transgenerational phenotype. Differently, transmission of epigenetic changes resulting from exposure to an environmental factor in males is limited to the $F_{0}$ and $\mathrm{F}_{1}$ generations because their somatic and germ line cells are the only ones that can be affected in this sex.

The impact of diet-induced maternal obesity on adiposity and metabolism in the offspring is quite well established, but the possible contribution of obese fathers is not clear at all (Scarino 2008; Harris and Seckl 2011; Youngson and Whitelaw 2008). However, increasing evidence indicates that fathers have an important biological role in obesity and in metabolic programming of their offsprings, and that epigenetic regulation of gene expression is critical during spermatogenesis. It has already been demonstrated that obesity affects sperm concentration and their motility and in humans increases DNA damage (Zamudio et al. 2008).

It has been reported that male mice whose mothers consumed a high fat diet (HFD) were heavier, insulinresistant, and diabetic and that produced a second generation of insulin-resistant but not obese animals (Dunn and Bale 2009). It is not known whether their insulin resistance is the result of paternal in utero exposure or of their adult sequelae of obesity and diabetes. These results indicate that fathers can transmit obesity and metabolic diseases intergenerationally, either directly or indirectly like through exposure to a HFD. A recent letter published in Nature by $\mathrm{Ng}$ et al. (2010) reports that in Sprague-Dawley rats paternal chronic exposure to HFD affects $\beta$-cell function in $F_{1}$ female offspring. HFD consumption by $F_{0}$ fathers was not associated with changes in litter size or sex ratio, but significantly altered the expression of a number of genes expressed by pancreatic islets and genes affecting several networks and cellular pathways in adult female offspring. When males were fed a HFD while females consumed a control diet, body weight, adiposity, and plasma leptin of the litter increased, and the animals became glucose intolerant and resistant to insulin. Compared to controls, their female offspring had normal adiposity but developed an early onset of impaired insulin secretion and glucose tolerance that worsened with time and developed a diabetes-like condition in adulthood. Paternal HFD exposure led to reduced relative islet area and $\beta$-cell area in the offspring, implying impaired $\beta$-cell replication. An increase in small islets was also observed in the offspring of HFD fathers as a compensatory response to maintain normal $\beta$-cell mass. Adult female offspring whose fathers were fed HFD displayed an altered expression of 642 genes in pancreatic islet. These genes belonged to 13 functional clusters, including cation and ATP binding, cytoskeleton, and intracellular transport. The molecular networks identified were consistent with the alterations in pancreas morphology and indicated impaired insulin-granule exocytosis. The results represent the first direct demonstration in any species that a paternal environmental exposure, HFD consumption, can induce intergenerational transmission of impaired glucose-insulin homeostasis in their female offspring.

In humans, it is known that paternal insulin resistance/ diabetes is inversely associated with offspring birthweight and increase subsequent risk of diabetes (Wannamethee et al. 2004). Although genetic factors may contribute, the available data show that paternal exposure to HFD can induce a similar phenotype in offspring. It has not yet been determined whether similar defects occur also in male offspring. Paternal lifestyle and specific environmental factors can affect spermatogenesis at the level of germ and Sertoli cells as well as of seminal fluid composition. Increased testicular temperature as a result of fat accumulation due to high dietary fat consumption can be genotoxic to germ cells, leading to increased DNA damage through oxidative injury. Moreover, hyperleptinaemia, hyperinsulinaemia, and the resulting hypogonadotrophic hypogonadism in obese males could affect spermatogenesis. A HFD may also interfere with Sertoli cell proliferation and on DNA reprogramming of gametes. The paper by $\mathrm{Ng}$ et al. (2010) is the first direct demonstration that a paternal 
environmental exposure like HFD consumption induces intergenerational transmission of impaired glucose-insulin homeostasis in female daughters. The mechanisms involved include epigenetic modifications whose functional implications are not fully understood. The results reported by the authors represent an example of multigenerational exposures. The father ( $\mathrm{F}_{0}$ generation) is directly exposed, as is his sperm, which will generate the $F_{1}$ offspring. Similarly, exposure of a pregnant female to an environmental factor can affect herself, the $F_{1}$ generation fetus that she carries, as well as the fetus' germ line that will then generate an $\mathrm{F}_{2}$ offspring. Multigenerational exposures involving a somatic cell-mediated effect on the $F_{0}$ generation and a potentially epigenetic effect on the germ line of the $F_{1}$ generation could therefore indirectly promote generational phenotypes such as the adult onset of disease in subsequent generations. However, more studies are required to clarify this aspect. It remains to be determined whether the effect of environmental exposure of the $\mathrm{F}_{0}$ generation fathers on the $F_{1}$ generation daughters is transgenerational and transmitted to the $\mathrm{F}_{2}$ generation, and it should also be more thoroughly investigated whether adult exposure can promote an epigenetic transgenerational inheritance. These findings demonstrate a paternal role in the early life origins of some diseases that could amplify the diabetes epidemics.

To test whether transgenerational inheritance of environmental information occurs in mammals, a group of US and Israeli scientists carried out an expression profiling screen for genes, responding to paternal diet in mice (Carone et al. 2010). The offspring of males fed a lowprotein diet exhibited elevated hepatic expression of several genes involved in lipid and cholesterol biosynthesis, with increased level of cholesterol and decreased level of cholesterol esters, relative to the offspring of males fed a control diet. Epigenomic profiling of offspring livers identified numerous small (no more than $20 \%$ ) changes in cytosine methylation at a putative enhancer for the key lipid transcription peroxisome proliferator-activated receptor alpha $(\operatorname{PPAR} \alpha)$, which is associated with the father's diet. These changes correlated also with the downregulation of the $\operatorname{PPAR} \alpha$ gene in the offspring. However, the authors did not find any effects of the paternal diet on methylation of the PPAR $\alpha$ locus in sperm, as overall sperm cytosine methylation pattern was largely conserved under various dietary regimes. These results identified a set of physiological pathways whose expression is sensitive to paternal diet. Specifically, the authors have found that hepatic expression of genes involved in proliferation and in cholesterol biosynthesis can be regulated by paternal diet, and that these changes are reflected in the levels of several lipid metabolites. Combined with the study showing that offspring glucose levels are affected by paternal fasting in mice (Anderson et al. 2006), these results demonstrate that in rodents, paternal diet exerts wide-ranging effects on metabolism in the offspring.

The implications of the results are many and important and would require a more in-depth discussion. In fact, the inheritance and transmission of acquired characters induced by environmental factors like the diet can be considered Lamarckian inheritance where organisms can inform their progeny about prevalent environmental conditions. The results of the two papers ( $\mathrm{Ng}$ et al. 2010; Carone et al. 2010) represent the first direct demonstration that paternal diet can induce transgenerational epigenetic reprogramming of gene expression in mammals that may have relevant implications for human health. Even though these and other observations indicate but do not necessarily demonstrate a Lamarck-like mechanism of evolution, it should be reconsidered the complexity of the environmentgenome relationship under a different perspective.

\section{References}

Anderson LM, Riffle L, Wilson R, Travlos GS, Lubomirski MS, Alvord WG (2006) Preconceptional fasting of fathers alters serum glucose in offspring of mice. Nutrition 22:327-331

Carone BR, Fauquier L, Habib N, Shea JM, Hart CE, Li R, Bock C, Li C, Gu H, Zamore PD, Meissner A, Weng Z, Hofmann HA, Friedman N, Rando OJ (2010) Paternally induced transgenerational environmental reprogramming of metabolic gene expression in mammals. Cell 143:1084-1096

Dunn GA, Bale TL (2009) Maternal high-fat diet promotes body length increases and insulin insensitivity in second generation mice. Endocrinology 150:4999-5009

Graham TE, Kahn BB (2007) Tissue specific alterations of glucose transport and molecular mechanisms of intertissue communication in obesity and type 2 diabetes. Horm Metab Res 39:717-721

Harris A, Seckl J (2011) Glucocorticoids, prenatal stress and the programming of disease. Horm Behav 59:279-289

Kahn BB, Flier JS (2000) Obesity and insulin resistance. J Clin Invest 106:473-481

Ng SF, Lin RCY, Laybutt DR, Barres R, Owens JA, Morris MJ (2010) Chronic high-fat diet in fathers programs $\beta$-cell dysfunction in female rat offspring. Nature 467:963-966

Reaven GM (1995) Pathophysiology of insulin resistance in human disease. Physiol Rev 75:473-486

Scarino ML (2008) A sideways glance. Do you remember your grandmother's food? How epigenetic changes transmit consequences of nutritional exposure from one generation to the next. Genes Nutr 3:1-3

Wannamethee SG, Lawlor DA, Whincup PH, Walker M, Ebrahim S, Davey-Smith G (2004) Birthweight of offspring and paternal insulin resistance and paternal diabetes in late adulthood: cross sectional survey. Diabetologia 47:12-18

Youngson NA, Whitelaw E (2008) Transgenerational epigenetic effects. Ann Rev Genomics Hum Genet 9:233-257

Zamudio NM, Chong S, O'Bryan MK (2008) Epigenetic regulation in male germ cells. Reproduction 136:131-146 